\title{
Reliability of Synthetic Brain MRI for Assessment of Ischemic Stroke with Phantom Validation of a Relaxation Time Determination Method
}

\author{
Chia-Wei Li ${ }^{1,+}{ }^{\text {, Ai-Ling Hsu }}{ }^{1, \dagger}$, Chi-Wen C. Huang ${ }^{1,2}$, Shih-Hung Yang ${ }^{1,2} \mathbb{D}^{-}$, Chien-Yuan Lin ${ }^{3}$, \\ Charng-Chyi Shieh ${ }^{3}$ and Wing P. Chan ${ }^{1,2,4, *}$ \\ 1 Department of Radiology, Wan Fang Hospital, Taipei Medical University, Taipei 116, Taiwan; \\ chiawei.lee@gmail.com (C.-W.L.); irene751110@gmail.com (A.-L.H.); cth.mail05@gmail.com (C.-W.C.H.); \\ schpeltor_tw@yahoo.com.tw (S.-H.Y.) \\ 2 Department of Radiology, School of Medicine, College of Medicine, Taipei Medical University, \\ Taipei 110, Taiwan \\ 3 GE Healthcare, Taipei 104, Taiwan; Eddy.Lin@ge.com (C.-Y.L.); mark.shieh@ge.com (C.-C.S.) \\ 4 Medical Innovation Development Center, Wan Fang Hospital, Taipei Medical University, Taipei 116, Taiwan \\ * Correspondence: wingchan@tmu.edu.tw; Tel.: +886-2-2930-7930 (ext. 1300); Fax: +886-2-2931-6809 \\ + These authors contributed equally to this work.
}

Received: 10 May 2020; Accepted: 10 June 2020; Published: 14 June 2020

\begin{abstract}
The reliability of relaxation time measures in synthetic magnetic resonance images (MRIs) of homemade phantoms were validated, and the diagnostic suitability of synthetic imaging was compared to that of conventional MRIs for detecting ischemic lesions. Phantoms filled with aqueous cupric-sulfate $\left(\mathrm{CuSO}_{4}\right)$ were designed to mimic spin-lattice $\left(\mathrm{T}_{1}\right)$ and spin-spin $\left(\mathrm{T}_{2}\right)$ relaxation properties and were used to compare their accuracies and stabilities between synthetic and conventional scans of various brain tissues. To validate the accuracy of synthetic imaging in ischemic stroke diagnoses, the synthetic and clinical scans of 18 patients with ischemic stroke were compared, and the quantitative contrast-to-noise ratios (CNRs) were measured, using the Friedman test to determine significance in differences. Results using the phantoms showed no significant differences in the interday and intersession synthetic quantitative $T_{1}$ and $T_{2}$ values. However, between synthetic and referenced $\mathrm{T}_{1}$ and $\mathrm{T}_{2}$ values, differences were larger for longer relaxation times, showing that image intensities in synthetic scans are relatively inaccurate in the cerebrospinal fluid (CSF). Similarly, CNRs in CSF regions of stroke patients were significantly different on synthetic $\mathrm{T}_{2}$-weighted and $\mathrm{T}_{2}$-fluid-attenuated inversion recovery images. In contrast, differences in stroke lesions were insignificant between the two. Therefore, interday and intersession synthetic $T_{1}$ and $T_{2}$ values are highly reliable, and discrepancies in synthetic $T_{1}$ and $T_{2}$ relaxation times and image contrasts in CSF regions do not affect stroke lesion diagnoses. Additionally, quantitative relaxation times from synthetic images allow better estimations of ischemic stroke onset time, consequently increasing confidence in synthetic MRIs as diagnostic tools for ischemic stroke.
\end{abstract}

Keywords: brain; magnetic resonance imaging; technology; white matter

\section{Introduction}

In synthetic magnetic resonance images (MRIs), multiple sets of sequence properties are used to obtain quantitative values for proton density, longitudinal relaxation time $\left(\mathrm{T}_{1}\right)$, and transverse relaxation time $\left(\mathrm{T}_{2}\right)$ at each imaging voxel. By adjusting the imaging parameters repetition time (TR), echo time (TE), and inversion time (TI), a technologist can mathematically produce multiple image contrast weightings from a single synthetic scan [1-3] once the quantitative values for $T_{1}, T_{2}$, and proton density 
are generated. Synthetic MRI technology using a two-dimensional fast spin-echo (SE) multi-dynamic, multi-echo sequence was recently approved by the US Food and Drug Administration and has also been used in clinical brain studies [4]. Synthetic MRIs allow completion of diagnostic brain examinations in about 4 to $7 \mathrm{~min}$ using a single scan. The shorter scan time is beneficial to stroke patients because it increases the diagnostic procedure's efficiency and comfort. Synthetic MRIs also provide a distinct tissue relaxation time [5-7], enabling a more accurate estimation of ischemic stroke status [8,9]. Because establishing an optimum relaxation time is a prerequisite for generating a synthetic image, achieving favorable synthetic image contrast depends on the accuracy of that value.

This study was conducted to validate the reliabilities of $T_{1}$ and $T_{2}$ relaxation times obtained from synthetic MRIs, and to investigate the achievability of sufficient contrast between stroke lesions and normal brain tissues using synthetic MRIs.

\section{Materials and Methods}

Imaging was performed using a 3T clinical scanner (MR750w; GE Healthcare, Milwaukee, WI, USA) with a body coil as the transmitter and a 24-channel array head coil as the receiver. The research protocol was approved by the X Medical University Joint Institutional Review Board (No. N201706024). All participants gave written informed consent.

\subsection{Synthetic MRI Technique}

Synthetic scans were obtained using MRI compilation (MAGiC) software and one multi-dynamic multi-echo sequence with the following parameters: repetition time (TR), $4123 \mathrm{~ms}$; TE, 21.4 and $96.3 \mathrm{~ms}$; and echo-train length, 14 echoes. Shared parameters across all scans were: matrix size, $256 \times 256$; slice thickness, $5 \mathrm{~mm}$; slice gap, $1 \mathrm{~mm}$; frequency, 20 slices per scan; and field of view, $160 \times 160 \mathrm{~mm}$ for phantom scans and $192 \times 192 \mathrm{~mm}$ for brain scans. Scan time was about $4 \mathrm{~min}$.

\subsection{Specifically Designed Phantoms}

This study was conducted to validate the reliabilities of $\mathrm{T}_{1}$ and $\mathrm{T}_{2}$ relaxation times from synthetic MRIs of phantoms containing sealed tubes filled with specific concentrations of aqueous $\mathrm{CuSO}_{4}$ (anhydrous copper sulfate; $97.5 \%$ purity; Nacalai Tesque, Kyoto, Japan) and fixed in a plastic tank (Figure 1). Two phantoms were designed specifically to give $T_{1}$ and $T_{2}$ values between about 1100 and $2300 \mathrm{~ms}$ and 50 and $240 \mathrm{~ms}$, respectively [10], mimicking the primary components of the brain: white matter (WM), gray matter (GM), and cerebrospinal fluid (CSF) [11-14].

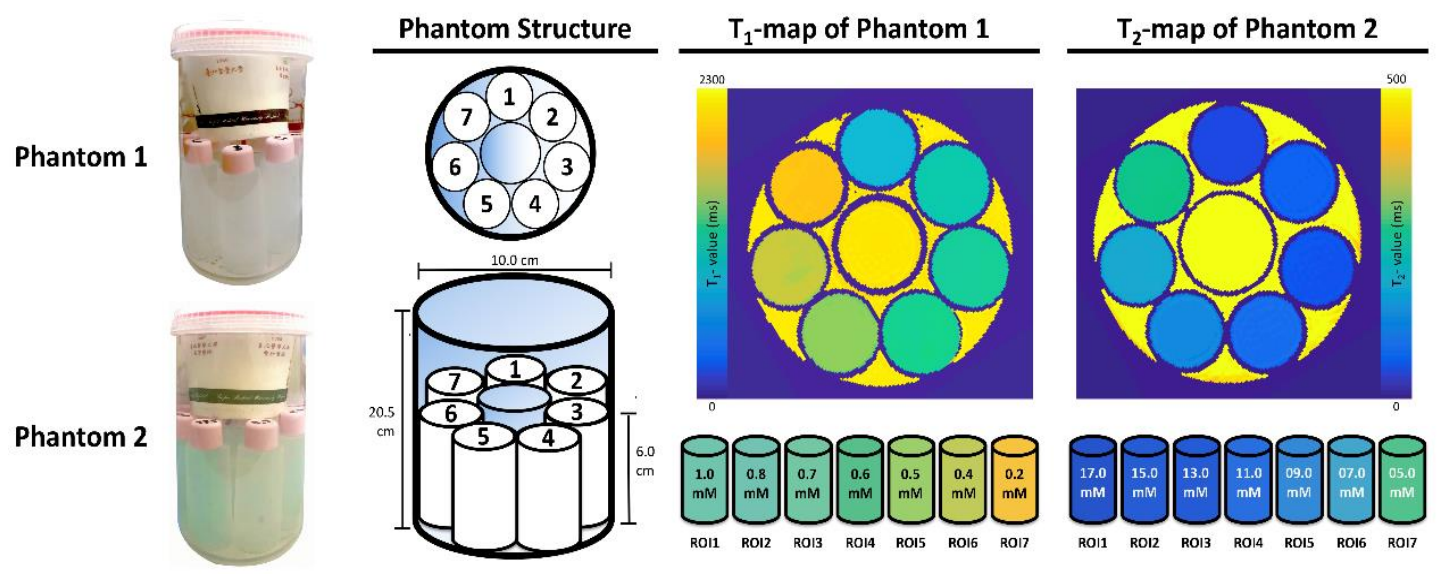

Figure 1. The structures of two phantoms designed for validating $T_{1}$ - and $T_{2}$-value quantitation. Plastic tubes (cylinder structure with $7.1 \mathrm{~cm}^{2}$ in dimension and $6.0 \mathrm{~cm}$ in height) filled with various concentrations of $\mathrm{CuSO}_{4}$ were sealed and fixed in the plastic tanks. The plastic tank was filled with normal saline to minimize image artifacts. 


\subsection{Phantom Evaluation}

The reference $\mathrm{T}_{1}$ value was quantified using a conventional $\mathrm{SE}$ with an inversion recovery sequence having the following parameters: TR, 10,000 ms; TE, $8.9 \mathrm{~ms}$; and a TI series $(100,300,500,700,800$, $900,1000,1100,1200,1300,1400,1500,1800,2400,3000$, and $4000 \mathrm{~ms}$ ). The reference $\mathrm{T}_{2}$ value was quantified using a multi-echo fast-SE sequence with the following parameters: TR, 10,000 ms, and a TE series $(9.9,19.9,29.8,39.7,49.6,59.6,69.5,79.4,89.4,99.3,109.2,119.2,129,139,149,158.8,168.7,178.6$, 188.5 , and $198.4 \mathrm{~ms}$ ). Both values from all scans were calculated using an in-house MATLAB script for nonlinear least-squares curve fitting (MathWorks, Natick, MA, USA) [15]. Interday reproducibility was assessed using the imaging coverage applied in conventional scans. Data were acquired from both phantoms on two days, one week apart, performing seven repetitions at 30-min intervals, and all synthetic data were used to assess intersession repeatability. The percent difference between the synthetic and conventional values was calculated as:

$$
\text { Difference }=\left(\left(\mathrm{T}_{\text {synthetic scan }}-\mathrm{T}_{\text {reference }}\right) / \mathrm{T}_{\text {reference }}\right) \times 100 \% \text {. }
$$

The differences obtained using the concentrations of aqueous $\mathrm{CuSO}_{4}$ in those two specific phantoms were calculated for quantitative validation.

\subsection{Participant and Diagnostic Score Evaluation}

Twenty patients with chronic stroke (>6 months), free of known neurologic and psychiatric diagnoses, were enrolled between 20 August 2017, and 28 December 2017, to explore the image contrasts in synthetic scans and to quantitatively compare synthetic and clinical images. Two were removed from the analysis due to excessive head movement. The mean (standard deviation, SD) of the resulting sample of 18 patients (14 men) was 59.6 (6.4) years.

Standardized stroke MRIs and synthetic scans were evaluated using double-blind testing. Two radiologists rated image quality on an 11-point scale ( 0 , entirely poor to 10 , entirely good) and answered one question: "Are these images acceptable for diagnostic use? (yes or no)." The display order of the patient images was randomly generated by MATLAB.

Images were prospectively acquired using a fixed set of parameters closely approximating a standardized stroke MRI protocol [16]. Images of clinical stroke were acquired first; then synthetic scans were obtained, including fast-SE with inversion-recovery axial plane $\mathrm{T}_{1}$-weighted imaging (TR, $1800 \mathrm{~ms}$; TE, $23 \mathrm{~ms}$; TI, $750 \mathrm{~ms}$ ), fast-SE coronal plane $\mathrm{T}_{2}$-weighted imaging (TR, $4545 \mathrm{~ms}$; TE, $110 \mathrm{~ms}$ ), and $\mathrm{T}_{2}$-fluid-attenuated inversion recovery (FLAIR) imaging (TR, $9000 \mathrm{~ms}$; TE, 92 ms; TI, $2472 \mathrm{~ms}$ ). One echo-planar-imaging-based diffusion-weighted image (TR, $6000 \mathrm{~ms}$; TE, $77 \mathrm{~ms}$ ) was also acquired, using $1000 \mathrm{~s} / \mathrm{mm}^{2}$ for the b-value, and one experienced radiologist located the ischemic lesion in each patient (Table 1).

Table 1. Demographic data of each included stroke patient.

\begin{tabular}{cccc}
\hline Patient $(\boldsymbol{n}=\mathbf{1 8})$ & Age (Year) & Sex & Lesion Location(s) \\
\hline Patient 01 & 64 & Male & Left striatum \\
\hline Patient 02 & 44 & Female & Left striatum \\
\hline Patient 03 & 64 & Male & Left cerebellum \\
\hline Patient 04 & 66 & Male & Left striatum \\
\hline Patient 05 & 63 & Female & Right temporal and frontal lobes \\
\hline Patient 06 & 50 & Male & Left parietal lobe \\
\hline Patient 07 & 66 & Male & Left frontal lobe \\
\hline Patient 08 & 55 & Male & Left striatum \\
\hline Patient 09 & 56 & Male & Left parietal lobe
\end{tabular}


Table 1. Cont.

\begin{tabular}{cccc}
\hline Patient $(\boldsymbol{n}=\mathbf{1 8})$ & Age (Year) & Sex & Lesion Location(s) \\
\hline Patient 10 & 56 & Female & Left insula \\
\hline Patient 11 & 58 & Female & Right parietal lobe \\
\hline Patient 12 & 69 & Male & Left striatum \\
\hline Patient 13 & 55 & Male & Right striatum \\
\hline Patient 14 & 62 & Male & Left cerebellum \\
\hline Patient 15 & 57 & Male & Left parietal lobe and right striatum \\
\hline Patient 16 & 57 & Male & Left striatum \\
\hline Patient 17 & 66 & Male & Right striatum \\
\hline Patient 18 & 64 & Male & Right parietal lobe
\end{tabular}

Preprocessing, analysis, and spatial segmentation were applied to all datasets, and mean image intensities for WM, GM, and CSF were extracted. The ICBM-152 [17,18] and Anatomical Automatic Labeling templates $[19,20]$ were normalized into individual images of each patient, and the mean signal intensity of the bilateral thalamus was extracted for image contrast standardization (Figure 2). The standardized image contrast-to-noise ratio (CNR) was calculated as:

\section{$\mathrm{CNR}=($ mean intensity of WM/GM/CSF/stroke lesion - mean intensity of thalamus)/standard deviation of thalamus.}

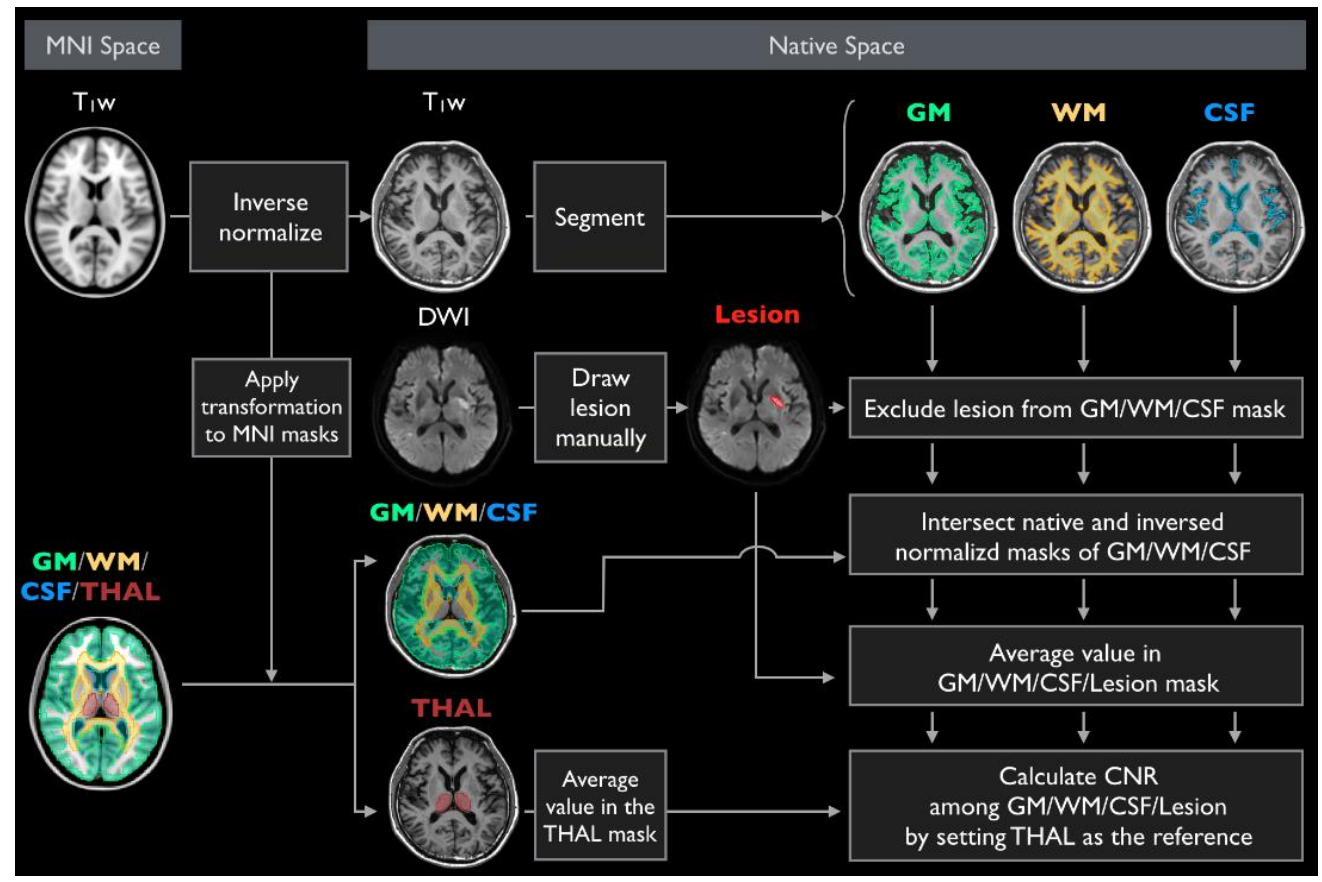

Figure 2. Data processing flowchart for quantitative tissue contrast validating synthetic scans.

\subsection{Statistical Analyses}

Statistical analyses were performed using the R software program with $t$ test functionality, version 3.5.1 (R Core Team 2018. R: A language and environment for statistical computing. R Foundation for Statistical Computing, Vienna, Austria. URL http://www.R-project.org/). The Friedman test was used to measure interday and intersession repeatabilities and the image contrast between clinical and 
synthetic images, allowing quantitative validation of phantom and patient data. A difference was considered statistically significant when the Bonferroni-corrected $p$-value was $<0.05$.

\section{Results}

Patient demographic data and the clinical locations of the stroke lesions are shown in Table 1.

\subsection{Phantom Evaluation}

Table 2 shows the interday and intersession quantitative $\mathrm{T}_{1}$ and $\mathrm{T}_{2}$ values across all applicable concentrations of aqueous $\mathrm{CuSO}_{4}$, acquired using synthetic scanning. The Friedman test showed no significant differences between the interday and intersession values. The reference $T_{1}$ and $T_{2}$ values were obtained using conventional SE methods. Differences in $\mathrm{T}_{1}$ and $\mathrm{T}_{2}$ between the synthetic and conventional scans are shown in Figure 3. Synthetic $\mathrm{T}_{1}$ values were closer to the reference values when $\mathrm{CuSO}_{4}$ concentrations were higher (at 0.7 to $1.0 \mathrm{mmol} / \mathrm{L}$, the difference was $5-8 \%$, whereas at $<0.2 \mathrm{mmol} / \mathrm{L}$, the difference was $>20 \%$ ). A similar trend was seen for synthetic $\mathrm{T}_{2}$ values (at 11.0 to $20.0 \mathrm{mmol} / \mathrm{L}$, the difference was $<10 \%$, whereas at 7.0 to $9.0 \mathrm{mmol} / \mathrm{L}$, the difference was $>20 \%$ ).
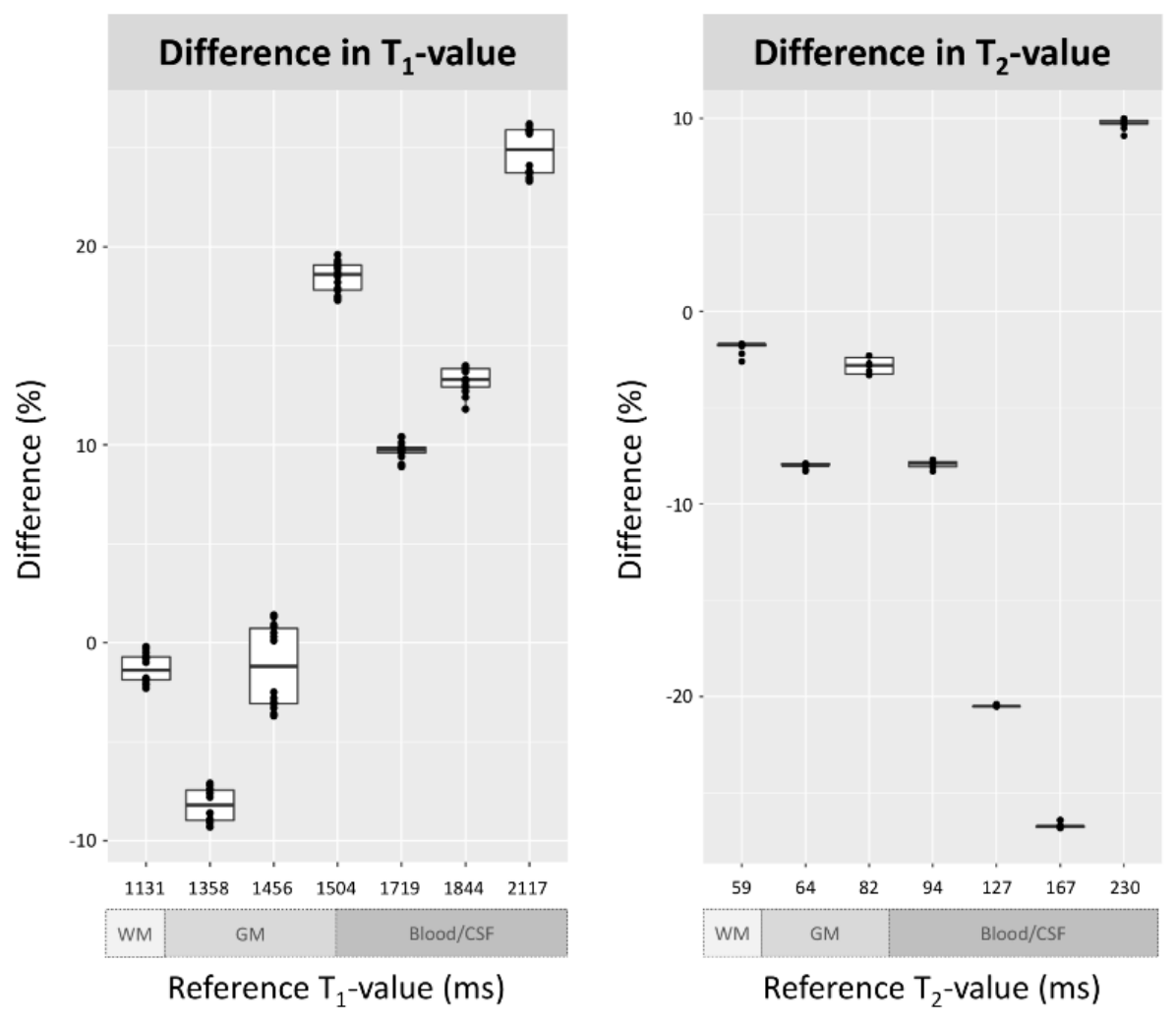

Figure 3. The difference in $\mathrm{T}_{1}$ - and $\mathrm{T}_{2}$-values of two aqueous $\mathrm{CuSO}_{4}$ phantoms between the synthetic and clinical scans. 
Table 2. Average quantitative values acquired using conventional magnetic resonance imaging (MRI) spin-echo sequences and synthetic MRI scans.

\begin{tabular}{|c|c|c|c|c|c|c|c|c|c|c|c|c|c|c|c|}
\hline \multicolumn{16}{|c|}{$\mathrm{T}_{1}$ Value } \\
\hline \multirow{2}{*}{$\underset{\text { Concentration }}{\mathrm{CuSO}_{4}}$} & \multirow{2}{*}{$\begin{array}{l}\text { Reference } \\
\text { Value }\end{array}$} & \multicolumn{7}{|c|}{ Quantitative Value, Synthetic Scan, Day 1} & \multicolumn{7}{|c|}{ Quantitative Value, Synthetic Scan, Day 2} \\
\hline & & 1-1 & $1-2$ & $1-3$ & $1-4$ & $1-5$ & $1-6$ & $1-7$ & $2-1$ & $2-2$ & $2-3$ & $2-4$ & $2-5$ & $2-6$ & $2-7$ \\
\hline $1.0 \mathrm{mM}$ & 1131.5 & 1123.1 & 1128.8 & 1122.6 & 1127.7 & 1122.2 & 1119.9 & 1126.2 & 1110.5 & 1111.0 & 1111.1 & 1105.6 & 1107.5 & 1111.2 & 1107.8 \\
\hline $0.8 \mathrm{mM}$ & 1357.8 & 1254.1 & 1254.9 & 1257.5 & 1260.1 & 1252.1 & 1262.0 & 1257.9 & 1240.8 & 1241.3 & 1231.0 & 1231.7 & 1236.6 & 1235.1 & 1233.9 \\
\hline $0.7 \mathrm{mM}$ & 1456.4 & 1463.0 & 1474.8 & 1476.3 & 1470.0 & 1460.8 & 1468.6 & 1457.3 & 1419.8 & 1416.0 & 1411.2 & 1402.4 & 1412.8 & 1407.9 & 1404.4 \\
\hline $0.6 \mathrm{mM}$ & 1503.7 & 1785.4 & 1798.6 & 1794.5 & 1790.5 & 1787.7 & 1781.8 & 1793.0 & 1789.3 & 1776.9 & 1767.5 & 1764.9 & 1771.9 & 1772.5 & 1764.5 \\
\hline $0.5 \mathrm{mM}$ & 1719.0 & 1883.3 & 1892.6 & 1889.5 & 1885.2 & 1879.7 & 1874.3 & 1898.2 & 1887.6 & 1884.6 & 1872.3 & 1887.2 & 1897.6 & 1888.2 & 1885.8 \\
\hline $0.4 \mathrm{mM}$ & 1843.8 & 2071.6 & 2088.3 & 2086.3 & 2082.1 & 2077.3 & 2061.3 & 2084.3 & 2099.5 & 2095.6 & 2088.2 & 2096.7 & 2101.7 & 2099.8 & 2100.5 \\
\hline $0.2 \mathrm{mM}$ & 2117.2 & 2612.3 & 2621.5 & 2620.8 & 2620.0 & 2614.7 & 2610.0 & 2628.2 & 2663.2 & 2665.0 & 2662.4 & 2665.8 & 2669.1 & 2666.3 & 2671.7 \\
\hline \multicolumn{16}{|c|}{$T_{2}$ Value } \\
\hline \multirow{2}{*}{$\underset{\text { Concentration }}{\mathrm{CuSO}_{4}}$} & \multirow{2}{*}{$\begin{array}{l}\text { Reference } \\
\text { Value }\end{array}$} & \multicolumn{7}{|c|}{ Quantitative Value, Synthetic Scan, Day 1} & \multicolumn{7}{|c|}{ Quantitative Value, Synthetic Scan, Day 2} \\
\hline & & $1-1$ & $1-2$ & $1-3$ & $1-4$ & $1-5$ & $1-6$ & $1-7$ & $2-1$ & $2-2$ & $2-3$ & $2-4$ & $2-5$ & $2-6$ & $2-7$ \\
\hline $20.0 \mathrm{mM}$ & 58.5 & 57.4 & 57.5 & 57.5 & 57.5 & 57.4 & 57.4 & 56.9 & 57.4 & 57.5 & 57.5 & 57.5 & 57.4 & 57.4 & 57.2 \\
\hline $15.0 \mathrm{mM}$ & 64.0 & 58.9 & 58.9 & 58.9 & 58.9 & 58.9 & 58.9 & 58.7 & 58.9 & 58.9 & 58.9 & 58.9 & 58.9 & 58.9 & 58.7 \\
\hline $13.0 \mathrm{mM}$ & 82.4 & 80.5 & 80.6 & 80.1 & 80.1 & 79.7 & 79.7 & 80.2 & 80.5 & 80.6 & 80.1 & 80.1 & 79.7 & 79.7 & 79.9 \\
\hline $11.0 \mathrm{mM}$ & 94.0 & 86.4 & 86.6 & 86.6 & 86.2 & 86.7 & 86.7 & 86.8 & 86.4 & 86.6 & 86.6 & 86.2 & 86.7 & 86.7 & 86.5 \\
\hline $9.0 \mathrm{mM}$ & 126.7 & 100.8 & 100.7 & 100.7 & 100.7 & 100.8 & 100.8 & 100.8 & 100.8 & 100.7 & 100.7 & 100.7 & 100.8 & 100.8 & 100.7 \\
\hline $7.0 \mathrm{mM}$ & 166.9 & 122.9 & 122.4 & 122.4 & 122.4 & 122.2 & 122.2 & 122.3 & 122.9 & 122.4 & 122.4 & 122.4 & 122.2 & 122.2 & 122.4 \\
\hline $5.0 \mathrm{mM}$ & 230.3 & 253.3 & 252.7 & 253.2 & 252.2 & 252.8 & 252.8 & 253.1 & 253.2 & 252.5 & 253.1 & 252.1 & 252.7 & 252.7 & 251.3 \\
\hline
\end{tabular}




\subsection{Participant Evaluation}

Table 3 shows the subjective ratings by two radiologists of the quality of the synthetic and clinical images and the suitability of synthetic images for stroke diagnosis. The clinical and synthetic images were identically rated for diagnostic suitability. However, the image quality rating, which exceeded 8 for synthetic $\mathrm{T}_{1}$ - and $\mathrm{T}_{2}$-weighted scans and exceeded 7.8 for $\mathrm{T}_{2}$-FLAIR synthetic scans, was significantly greater for the corresponding clinical scans (Bonferroni-corrected $p=0.05$ ). Figure 4 shows representative clinical and synthetic scans of a stroke patient, demonstrating that the contrasts are similar. Instead of visually evaluating the image contrast, the CNR was determined in WM, GM, CSF, and stroke lesions (Figure 5). Differences were not significant in any of the WM and GM regions (see Figure 5) but were significant in the CSF regions on $\mathrm{T}_{2}$-weighted and $\mathrm{T}_{2}$-FLAIR images (Bonferroni-corrected $p=0.05$, multiple-comparison correction). In the lesion regions, CNRs were not significantly different between images reconstructed from synthetic scans and those acquired using clinical scanning methods.

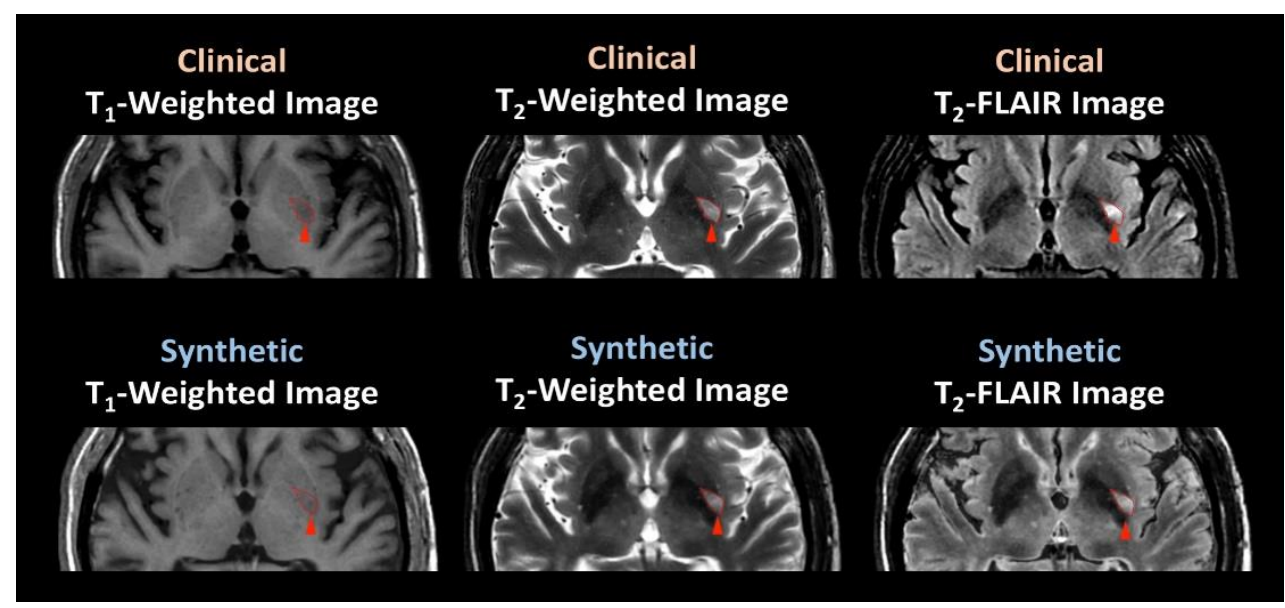

Figure 4. Images acquired by clinical and synthetic scanning of a 64-year-old male patient with stroke.
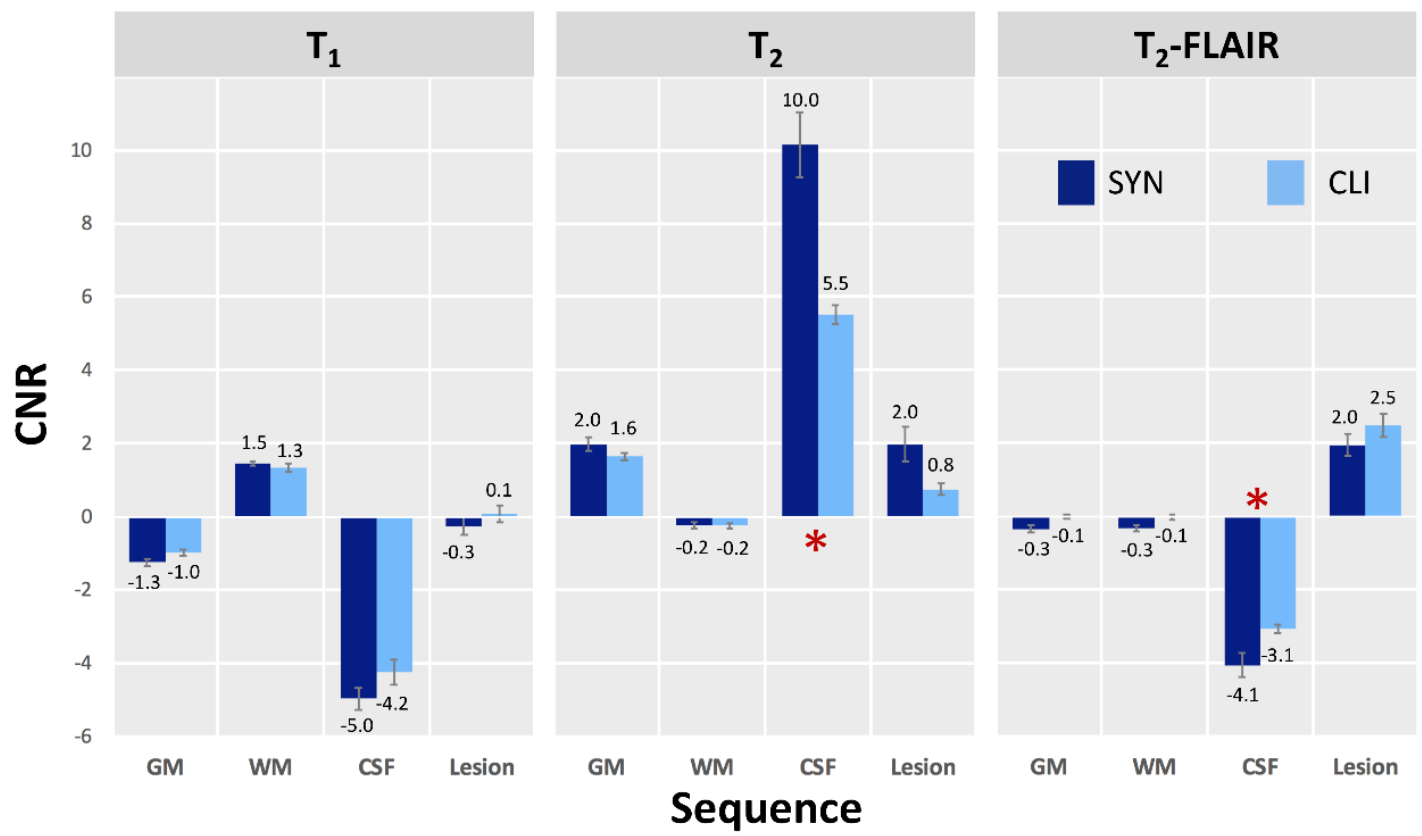

Figure 5. The standardized contrast-to-noise ratio (CNR) in synthetic and clinical scans of four brain regions. Significant differences were found between synthetic and clinical $\mathrm{T}_{2}$-weighted and $\mathrm{T}_{2}$-FLAIR images of cerebrospinal fluid regions ( ${ }^{*}$ Bonferroni corrected $p<0.05$ ). 
Table 3. Diagnostic image quality rated by two radiologists.

\begin{tabular}{ccccccc}
\hline \multirow{2}{*}{ Question } & \multicolumn{2}{c}{ T1-Weighted Image } & \multicolumn{2}{c}{ T2-Weighted Image } & \multicolumn{2}{c}{ T2-FLAIR Image } \\
\cline { 2 - 7 } & SYN & CLI & SYN & CLI & SYN & CLI \\
\hline $\begin{array}{c}\text { What is the image quality for } \\
\text { diagnostic use? a }\end{array}$ & $8.06^{* *}$ & 9.42 & $8.53^{*}$ & 9.42 & $7.89^{* *}$ & 9.36 \\
& $(0.79)$ & $(0.28)$ & $(1.00)$ & $(0.84)$ & $(1.94)$ & $(0.49)$ \\
\hline $\begin{array}{c}\text { Are these images acceptable } \\
\text { for diagnostic use? b }\end{array}$ & & & & 0.97 & 0.97 \\
\hline
\end{tabular}

Values are means, standard deviations are shown in parentheses. FLAIR = fluid-attenuated inversion recovery, SYN = synthetic scan, CLI = clinical scan. ${ }^{\text {a }}$ Eleven-point scale $(0-10) ;{ }^{b}$ Two-point scale $(0,1) ;{ }^{*}$ corrected $p<0.05$; ** corrected $p<0.01$.

\section{Discussion}

In this study, significant differences in $\mathrm{T}_{1}$ and $\mathrm{T}_{2}$ values across the 14 synthetic scans of the two phantoms were not found; therefore, the reliability of acquiring synthetic quantitative $T_{1}$ and $T_{2}$ values is high. The relaxation times varied across brain tissues, but smaller differences were found between the synthetic and reference values when they ranged from about 1131 to $1456 \mathrm{~ms}$ for $\mathrm{T}_{1}$ and from about 59 to $94 \mathrm{~ms}$ for $\mathrm{T}_{2}$. Differences were apparent in the CSF when $\mathrm{T}_{1}$ and $\mathrm{T}_{2}$ were greater than $1456 \mathrm{~ms}$ and $94 \mathrm{~ms}$, respectively. As seen with the phantoms, differences were large when relaxation times were in the range for CSF regions, confirming that results in CSF regions account for the differences found. Significant differences were not found between synthetic and clinical $\mathrm{T}_{2}$-FLAIR images of stroke lesions, showing that disparities in signal intensity from the various CSF regions did not affect the diagnosis of chronic stroke lesion, most commonly located in WM and GM. The ratings by two radiologists for diagnostic suitability concur with this result.

The literature demonstrates that the quantitative $T_{2}$ values of stoke lesions are significantly correlated with time since symptom onset [9]. Quantitative $T_{2}$ values are significantly higher for ischemic lesions than for healthy tissue, and using $T_{2}$ values to predict time since symptom onset is more accurate than using FLAIR images. One animal study [8] showed that quantitative values for $\mathrm{T}_{1}$ and $\mathrm{T}_{2}$ increased linearly with ischemia duration; in addition, a more accurate estimation of stroke duration was achieved using a pixel-by-pixel analysis to quantify relaxation times at a single point in time than by using signal intensity. Moreover, quantitative relaxation times provide additional information about tissue status in ischemia. Uncertainties in onset time estimates using quantitative $\mathrm{T}_{1}$ and $\mathrm{T}_{2}$ values vary from about 25 to $47 \mathrm{~min}$ in the first $5 \mathrm{~h}$ of ischemic stroke [21]. Therefore, in clinical images, estimating the onset time and identifying ischemic tissue are more accurate using quantitative relaxation times rather than signal intensities; they are free from operator bias, thereby increasing the reproducibility of time since symptom onset. However, they might not have a clinical application in diagnosing ischemic stroke because dozens of minutes are always required to quantify relaxation times using traditional clinical scanning methods. The synthetic technique uses the multi-dynamic, multi-echo sequence with four fixed inversion times and two echo times to simultaneously acquire quantitative values (including $\mathrm{T}_{1}, \mathrm{~T}_{2}$, and proton density) within a couple minutes. This efficiency allows quantitative relaxation times to be used for ischemia diagnoses.

Our results show no significant differences in stroke lesion regions between images reconstructed from synthetic $\mathrm{T}_{2}$-FLAIR scans and those acquired using clinical scans. However, the image quality ratings of the synthetic $T_{2}$-FLAIR scans were significantly lower than those for the corresponding clinical scans due to several artifacts in the former. These included granular hyperintensity signals occasionally appearing in the margins and flow artifacts [4,6], resulting from partial volume effects and flow effects not considered in the analytical signal model when estimating image parameters. Artifacts in synthetic $\mathrm{T}_{2}$-FLAIR scans resulted in lower image quality but showed little interference in diagnosing chronic stroke. Significant differences were also found between the synthetic and clinical $\mathrm{T}_{2}$-FLAIR images in the CSF regions; two radiologists found that the synthetic images were of lower quality, agreeing with the findings of others [4]. Two recent studies used deep learning algorithms to 
solve this problem. Hagiwara et al. attempted to improve the accuracy in brain CSF regions by using conditional generative adversarial network training to perform pixel-by-pixel translation methods, thus generating FLAIR images with contrasts closer to those of conventional FLAIR images [22]. The corrected synthetic FLAIR images provided Dice scores and image qualities that were closer to or more comparable with those found in conventional FLAIR images, and they showed fewer granular and swelling artifacts. Ryu et al. used 56 FLAIR datasets to train a deep-learning-based synthetic FLAIR method to correct for artifacts in synthetic FLAIR images [23], significantly improving the normalized root-mean-square values and the structural similarities. The results of these two studies suggest possible methods for correcting artifacts in synthetic images.

Our study has two limitations: the simple structure of our phantoms and the small sample size. First, in this study, we designed and used two phantoms with 14 specific concentrations of aqueous CuSO4, showing that the $\mathrm{T}_{1}$ - and $\mathrm{T}_{2}$-values are similar to those of brain tissue, thus validating the accuracy of quantitative relaxation times acquired by synthetic scanning. However, brain tissues are anisotropic structures and can affect relaxation processes, and the simple structure of our phantoms could not describe actual brain tissue but only changes in free fluid spaces. Some have demonstrated that other types of phantoms could better reflect the reality in brain tissue compared to ours [24-28]; therefore, we suggest using those phantoms in further studies validating quantitative relaxation times. Second, this infers that our results are not generalizable to other comparisons between synthetic and clinical scanning in chronic ischemic stroke. Therefore, these results cannot be applied to acute/sub-acute stroke or hemorrhagic stroke. Further investigations using images in acute, sub-acute, and hemorrhagic stroke are now necessary.

In summary, this study shows high interday and intersession reliabilities of quantitative relaxation times in synthetic scans. Although discrepancies in $\mathrm{T}_{1}$ and $\mathrm{T}_{2}$ relaxation times and image contrasts in CSF regions were observed in synthetic scans, they did not affect the diagnoses of stroke lesions. Also, quantitative measures of relaxation times by such a fast and efficient means can improve the accuracy of ischemic stroke assessment, consequently increasing confidence in applying synthetic MRIs to ischemic stroke diagnoses.

Author Contributions: C.-W.L., A.-L.H., and W.P.C. made substantial contributions to conception and study design; C.-W.L., A.-L.H., C.-W.C.H., and S.-H.Y. contributed to data acquisition, analysis, or interpretation; C.-Y.L. and C.-C.S. provided technical support; C.-W.L. composed the first draft. All authors made critical revisions related to important intellectual content, and all approved the final version for publication and agree to be accountable for all aspects of the work and will appropriately investigate and resolve questions related to the accuracy and integrity of any part of the work. All authors have read and agreed to the published version of the manuscript.

Funding: The authors gratefully acknowledge the receipt of partial financial support from the Inter-agency Research Fund, Wan Fang Hospital, Taipei Medical University (Grant No. 107-wf-swf-09).

Conflicts of Interest: Two co-authors, C.Y.L. and C.-C.S., are employees of GE Healthcare, Taiwan, and they provided sequence information and technical support for the MAGiC software. No funding was received from GE Healthcare for publication activities.

$\begin{array}{ll}\text { Abbreviations } \\ \text { CNR } & \text { contrast-to-noise ratio } \\ \text { CSF } & \text { cerebrospinal fluid } \\ \text { FLAIR } & \text { fluid-attenuated inversion recovery } \\ \text { GM } & \text { gray matter } \\ \text { MAGiC } & \text { magnetic resonance image compilation } \\ \text { SE } & \text { spin-echo } \\ \text { TE } & \text { echo time } \\ \text { TR } & \text { repetition time } \\ \text { TI } & \text { inversion time } \\ \text { WM } & \text { white matter }\end{array}$




\section{References}

1. Krauss, W.; Gunnarsson, M.; Andersson, T.; Thunberg, P. Accuracy and reproducibility of a quantitative magnetic resonance imaging method for concurrent measurements of tissue relaxation times and proton density. Magn. Reason. Imaging 2015, 33, 584-591. [CrossRef] [PubMed]

2. Warntjes, J.B.M.; Dahlqvist, O.; Lundberg, P. Novel method for rapid, simultaneous $T_{1}, T^{*}$, and proton density quantification. Magn. Reason. Med. 2007, 57, 528-537. [CrossRef] [PubMed]

3. Riederer, S.J.; Suddarth, S.A.; Bobman, S.A.; Lee, J.N.; Wang, H.Z.; MacFall, J.R. Automated MR image synthesis: Feasibility studies. Radiology 1984, 153, 203-206. [CrossRef]

4. Tanenbaum, L.N.; Tsiouris, A.J.; Johnson, A.N.; Naidich, T.P.; de Lano, M.C.; Melhem, E.R.; Quarterman, P.; Parameswaran, S.X.; Shankaranarayanan, A.; Goyen, M.; et al. Synthetic MRI for Clinical Neuroimaging: Results of the Magnetic Resonance Image Compilation (MAGiC) Prospective, Multicenter, Multireader Trial. AJNR Am. J. Neuroradiol. 2017, 38, 1103-1110. [CrossRef]

5. Granberg, T.; Uppman, M.; Hashim, F.; Cananau, C.; Nordin, L.E.; Shams, S.; Berglund, J.; Forslin, Y.; Aspelin, P.; Fredrikson, S.; et al. Clinical Feasibility of Synthetic MRI in Multiple Sclerosis: A Diagnostic and Volumetric Validation Study. AJNR Am. J. Neuroradiol. 2016, 37, 1023-1029. [CrossRef]

6. Blystad, I.; Warntjes, J.B.M.; Smedby, O.; Landtblom, A.-M.; Lundberg, P.; Larsson, E.-M. Synthetic MRI of the brain in a clinical setting. Acta Radiol. 2012, 53, 1158-1163. [CrossRef]

7. Warntjes, J.B.M.; Leinhard, O.D.; West, J.; Lundberg, P. Rapid magnetic resonance quantification on the brain: Optimization for clinical usage. Magn. Reason. Med. 2008, 60, 320-329. [CrossRef] [PubMed]

8. Rogers, H.J.; McGarry, B.L.; Knight, M.J.; Jokivarsi, K.T.; Gröhn, O.H.J.; Kauppinen, R.A. Timing the ischaemic stroke by 1 H-MRI: Improved accuracy using absolute relaxation times over signal intensities. NeuroReport 2014, 25, 1180-1185. [CrossRef] [PubMed]

9. Siemonsen, S.; Mouridsen, K.; Holst, B.; Ries, T.; Finsterbusch, J.; Thomalla, G.; Ostergaard, L.; Fiehler, J. Quantitative $\mathrm{T}_{2}$ values predict time from symptom onset in acute stroke patients. Stroke 2009, 40, 1612-1616. [CrossRef]

10. Thangavel, K.; Saritaş, E.Ü. Aqueous paramagnetic solutions for MRI phantoms at 3 T: A detailed study on relaxivities. Turk. J. Elect. Engineer. Comput. Sci. 2017, 25, 2108-2121. [CrossRef]

11. Lu, H.; Clingman, C.; Golay, X.; van Zijl, P.C.M. Determining the longitudinal relaxation time $\left(T_{1}\right)$ of blood at 3.0 Tesla. Magn. Reason. Med. 2004, 52, 679-682. [CrossRef]

12. Ethofer, T.; Mader, I.; Seeger, U.; Helms, G.; Erb, M.; Grodd, W.; Ludolph, A.; Klose, U. Comparison of longitudinal metabolite relaxation times in different regions of the human brain at 1.5 and 3 Tesla. Magn. Reason. Med. 2003, 50, 1296-1301. [CrossRef] [PubMed]

13. Greenman, R.L.; Shirosky, J.E.; Mulkern, R.V.; Rofsky, N.M. Double inversion black-blood fast spin-echo imaging of the human heart: A comparison between 1.5T and 3.0T. J. Magn. Reason. Imaging 2003, 17, 648-655. [CrossRef] [PubMed]

14. Gelman, N.; Gorell, J.M.; Barker, P.B.; Savage, R.M.; Spickler, E.M.; Windham, J.P.; Knight, R.A. MR imaging of human brain at $3.0 \mathrm{~T}$ : Preliminary report on transverse relaxation rates and relation to estimated iron content. Radiology 1999, 210, 759-767. [CrossRef] [PubMed]

15. Barral, J.K.; Gudmundson, E.; Stikov, N.; Etezadi-Amoli, M.; Stoica, P.; Nishimura, D.G. A robust methodology for in vivo $T_{1}$ mapping. Magn. Reason. Med. 2010, 64, 1057-1067. [CrossRef]

16. Schellinger, P.D.; Jansen, O.; Fiebach, J.B.; Hacke, W.; Sartor, K. A standardized MRI stroke protocol: Comparison with CT in hyperacute intracerebral hemorrhage. Stroke 1999, 30, 765-768. [CrossRef]

17. Fonov, V.; Evans, A.C.; Botteron, K.; Almli, C.R.; McKinstry, R.C.; Collins, D.L. Brain Development Cooperative Group. Unbiased average age-appropriate atlases for pediatric studies. Neuroimage 2011, 54, 313-327. [CrossRef] [PubMed]

18. Fonov, V.S.; Evans, A.C.; McKinstry, R.C.; Almli, C.R.; Collins, D.L. Unbiased nonlinear average age-appropriate brain templates from birth to adulthood. Neuroimage 2009, 47 (Suppl. 1), S102. [CrossRef]

19. Rolls, E.T.; Joliot, M.; Tzourio-Mazoyer, N. Implementation of a new parcellation of the orbitofrontal cortex in the automated anatomical labeling atlas. Neuroimage 2015, 122, 1-5. [CrossRef]

20. Tzourio-Mazoyer, N.; Landeau, B.; Papathanassiou, D.; Crivello, F.; Etard, O.; Delcroix, N.; Mazoyer, B.; Joliot, M. Automated anatomical labeling of activations in SPM using a macroscopic anatomical parcellation of the MNI MRI single-subject brain. Neuroimage 2002, 15, 273-289. [CrossRef] 
21. McGarry, B.L.; Jokivarsi, K.T.; Knight, M.J.; Gröhn, O.H.J.; Kauppinen, R.A. A Magnetic Resonance Imaging Protocol for Stroke Onset Time Estimation in Permanent Cerebral Ischemia. J. Vis. Exp. 2017, 16, e55277. [CrossRef] [PubMed]

22. Hagiwara, A.; Otsuka, Y.; Hori, M.; Tachibana, Y.; Yokoyama, K.; Fujita, S.; Andica, C.; Kamagata, K.; Irie, R.; Koshino, S.; et al. Improving the Quality of Synthetic FLAIR Images with Deep Learning Using a Conditional Generative Adversarial Network for Pixel-by-Pixel Image Translation. AJNR Am. J. Neuroradiol. 2019, 40, 224-230. [CrossRef] [PubMed]

23. Ryu, K.; Nam, Y.; Gho, S.-M.; Jang, J.; Lee, H.-J.; Cha, J.; Baek, H.J.; Jiyong, P.; Kim, D.H. Data-driven synthetic MRI FLAIR artifact correction via deep neural network. J. Magn. Reason. Imaging 2019, 50, 1413-1423. [CrossRef]

24. Krzyzak, A.; Klodowski, K.; Raszewski, Z. Anisotropic Phantoms in Magnetic Resonance Imaging. In Proceedings of the 37th Annual International Conference of the IEEE Engineering in Medicine and Biology Society (EMBC), Milan, Italy, 25-29 August 2015; pp. 414-417.

25. Kłodowski, K.; Krzyżak, A.T. Innovative anisotropic phantoms for calibration of diffusion tensor imaging sequences. Magn. Reason. Imaging 2016, 34, 404-409. [CrossRef] [PubMed]

26. Fieremans, E.; Lee, H.-H. Physical and numerical phantoms for the validation of brain microstructural MRI: A cookbook. Neuroimage 2018, 182, 39-61. [CrossRef]

27. Mazur, W.; Krzyzak, A.T.; Raszewski, Z. Towards the precise microstructural mapping. Testing new anisotropic phantoms with layered and capillary geometries. In Proceedings of the 41st Annual International Conference of the IEEE Engineering in Medicine and Biology Society (EMBC), Berlin, Germany, 23-27 July 2019; pp. 2835-2839.

28. De Souza, E.M.; Costa, E.T.; Castellano, G. Investigation of anisotropic fishing line-based phantom as tool in quality control of diffusion tensor imaging. Radiol. Phys. Technol. 2019, 12, 161-171. [CrossRef]

(C) 2020 by the authors. Licensee MDPI, Basel, Switzerland. This article is an open access article distributed under the terms and conditions of the Creative Commons Attribution (CC BY) license (http://creativecommons.org/licenses/by/4.0/). 